\title{
Autonomic dysreflexia during urodynamics
}

\author{
A Giannantoni, SM Di Stasi, G Scivoletto, A Mollo, A Silecchia, U Fuoco and G Vespasiani \\ IRCCS 'S. Lucia' Rehabilitation Hospital, Department of Surgery/Urology, 'Tor Vergata' University of Rome, \\ Rome, Italy
}

Autonomic dysreflexia (AD) is an acute syndrome characterised by inappropriate and massive autonomic response that occurs in patients with spinal cord injury above the T6 level.

Aims: to evaluate the incidence of AD during cystometry and the relationships with clinical and urodynamic features.

Patients and methods: Forty-eight spinal cord injury patients were studied by neurological and urological examination and urodynamic evaluation with concurrent recording of blood pressure, heart rate and symptoms and signs of AD. Patients were considered to have AD if blood pressure reached values higher than $150 / 100 \mathrm{mmHg}$.

Results: All the patients showed a significant increase of both systolic and diastolic blood pressure, although only 20 showed pressure values higher than $150 / 100 \mathrm{mmHg}$ (in seven of them without $\mathrm{AD}$ symptoms). AD was more frequent in cervical patients $(P=0.034)$, but did not correlate with any other clinical features: sex ratio, age, disease duration, completeness of lesion, incidence of detrusor hyperreflexia/areflexia and detrusor-sphincter dyssynergia, voiding modalities, usage of anticholinergic drugs. In three patients blood pressure increase began when uninhibited contraction started, in 11 it was coincident with unhibited contraction peak and in the other six it appeared at maximum bladder capacity.

Conclusions: (1) during urodynamic evaluation all the patients with lesion level above T6 showed signs of sympathetic stimulation, although only some showed dangerous blood pressure values; (2) the relationship between urodynamic data and dysreflexia crisis shows that both the presence of detrusor uninhibited contractions and bladder distension are able to stimulate the crisis; (3) treatment with anticholinergic drugs is not sufficient to prevent autonomic dysreflexia starting from the bladder, unless it induces detrusor areflexia. These patients are at risk of developing autonomic dysreflexia following bladder distension.

Keywords: Spinal cord injury; autonomic dysreflexia; urodynamics

\section{Introduction}

Autonomic dysreflexia (AD) is an acute syndrome characterised by inappropriate and massive autonomic response that occurs in patients with a spinal cord injury above the major sympathetic splanchnic outflow, usually at or above T6 level. ${ }^{1}$

Practically any cutaneous or visceral stimulus below the lesion, usually bladder and rectal distension, can lead a sudden reflex sympathetic discharge. ${ }^{1,2}$ It can occur spontaneously (chronic AD) or during diagnostic and therapeutic manoeuvres such as cystoscopy, cystometry, cystography, electroejaculation, penile vibration, enemas. Subjective symptoms are characterised by nausea, anxiety, malaise, a prickling sensation in the skull region and throbbing headache. The objective signs include light sweating and blushing, piloerection, increasing spasticity in all extremities, rise in blood pressure and compensatory bradycardia. Hypertension may cause seizures, intra-

Correspondence: A Giannantoni cerebral hemorrhage or death. Thus AD can lead to a severe emergency, which specialists and care personnel should be aware of and know how to manage. ${ }^{3}$

Furthermore, blood pressure can rise even without symptoms of autonomic dysreflexia; with regard to this phenomenon, previous studies demonstrated that urodynamics are helpful to detect significant blood pressure elevations during voiding in the absence of symptoms of autonomic dysreflexia. ${ }^{4}$

The relationships between $\mathrm{AD}$ and bladder function are not clear: some authors demonstrated that the increase in blood pressure may be caused by bladder distension during urodynamic evaluation, ${ }^{5}$ while others found that blood pressure increase was coincident with uninhibited detrusor contractions. ${ }^{4}$ In another study a correlation between blood pressure increase and the duration of uninhibited detrusor contractions was found. ${ }^{6}$

The aim of this study was to evaluate the incidence of AD during cystometry and the relationship with clinical and urodynamic features. 


\section{Patients and methods}

Between January 1995 and March 1997 we examined 48 Spinal Cord injury (SCI) patients $(42 \mathrm{M}, 6 \mathrm{~F})$ with a lesion at the T6 level or above. Mean age was $31 \pm 12.8$ years, mean disease duration was $48 \pm 69$ months. Three patients were examined while still in the spinal shock phase (two of them already had a history of AD). Twenty-three patients had a lesion at the cervical level, the other 25 at the dorsal (above T6). All the patients underwent complete neurological examination according to American Spinal Injury Association (ASIA) criteria ${ }^{7}$ : 39 showed an ASIA impairment scale A lesion, the other nine an incomplete lesion.

With regard to voiding modalities 24 patients performed clean intermittent catheterization, three had indwelling catheter or suprapubic catheter, three voided spontaneously and the other 18 used suprapubic tapping. Eighteen patients had been under anticholinergic therapy for a mean of $9.4 \pm 7.4$ months. Twenty-one patients had a history of AD symptoms.

None of the patients were receiving medications known to interfere with blood pressure or autonomic nervous system.

Urodynamic investigation was performed in all patients, according to the standards of the International Continence Society ${ }^{8}$ except when specifically noted. Patients with actual urinary infection were also excluded. Urodynamic evaluation consisted of cystometry with water at $37^{\circ} \mathrm{C}$ and a filling rate of $50 \mathrm{ml}$ per minute through a $6 \mathrm{~F}$ double lumen catheter with the patients in the supine position. Abdominal pressure was measured with $10 \mathrm{~F}$ intrarectal balloon catheter. Pelvic floor electromyography was recorded during cystometry with a bipolar wire electrode in the urethral sphincter. Filling was stopped when the patients reported sensation of fullness or at the moment of urine leaking or when bladder filling reached $500 \mathrm{ml}$; and bladder filling was stopped if blood pressure reached dangerous levels (190/110). We decided to stop bladder filling at a volume of $500 \mathrm{ml}$ or less because none of our patients had a capacity higher than that value; furthermore we decided to respect the usual conditions of filling and voiding of the patients in real life (for example in patients performing clean intermittent catheterization we did not overcome the volumes usually achieved with catheterization). ${ }^{4}$

Patients were not on alpha-blocker medications nor Calcium-antagonists, Nifedipine $10 \mathrm{mg}$ tablets were always available if necessary. However, none of our patients actually needed to take Nifedipine. In fact in patients with AD crisis during urodynamics, blood pressure values decreased spontaneously as soon as bladder filling was stopped and bladder was emptied.

The presence of detrusor hyperreflexia, areflexia, and detrusor-sphincter dyssynergia as defined by the standards of the International Continence Society, ${ }^{8}$ was noted. Detrusor-sphincter dyssynergia was classi- fied into three classes according to Blaivas and Barbalias criteria. ${ }^{9}$ For patients with detrusor hyperreflexia leak point pressure was also noted.

Bladder compliance was defined as the bladder volume $v s$ intravesical pressure ratio $(\mathrm{ml} / \mathrm{cm}$ water); it was assumed as reduced if $<12.5 \mathrm{ml} / \mathrm{cm}$ water.

Systolic and diastolic blood pressure (BP) and pulse rate (PR) were recorded before starting the filling $\mathrm{CMG}$; the filling was then started and the same vital signs were monitored every 2 min and at the beginning of each uninhibited detrusor contraction. During urodynamic evaluation any sensation of bladder filling, voiding or symptoms of $\mathrm{AD}$ was registered. There is no consensus on the exact values at which the blood pressure becomes dangerous. A person with an injury at or above $\mathrm{T} 6$ would be expected to have a baseline blood pressure between 90 and $110 \mathrm{mmHg}$. Guttman et al. ${ }^{10}$ described an autonomic dysreflexia episode occuring when the systolic blood pressure reached $20-40 \mathrm{mmHg}$ above baseline. Nevertheless the Autonomic Dysreflexia Guideline Development Panel ${ }^{11}$ decided to adopt $150 \mathrm{mmHg}$ systolic pressure as the value at which pharmacological treatment should be considered. According to the Panel and to other authors ${ }^{4}$ the test was considered positive for AD when systolic BP reached 150 and/or diastolic BP $100 \mathrm{mmHg}$, also in the absence of other clinical manifestations.

Statistical analysis was performed as follows: contingency tables were assessed with chi-square test or Fisher's exact test; differences between means were calculated with Student's $t$-test. Significance was assumed if $P<0.05$.

\section{Results}

Twenty patients (41.6\% of our sample) showed autonomic dysreflexia during urodynamic. To study the relationships between $\mathrm{AD}$ and clinical and cystometric features, patients were divided in two groups according to the presence of significant $\mathrm{AD}$ : group A patients without AD and group B patients with dysreflexia. The features of the two groups are shown in Table 1.

Group A consisted of 28 patients (58.4\%). With regard to voiding modalities, 16 patients used clean intermittent catheterization, nine suprapubic tapping, one voided spontaneously and two had indwelling catheter. Only eight patients had a history of AD symptoms; 11 patients were under therapy with anticholinergic drugs.

Group B consisted of 20 patients $(41.6 \%)$. In this group eight patients were under clean intermittent catheterization regimen, nine voided by means of suprapubic tapping, two voided spontaneously and one had suprapubic catheter. Seven patients were under anticholinergic drugs. Sixteen of these patients reported a history of AD symptoms. Urodynamic findings of the two groups are summarized in Table 2. 


\section{Statistical evaluation}

In group A mean systolic BP varied from $112.1 \pm 13.3 \mathrm{mmHg}$ (mean + standard deviation) in basal conditions to $121.6 \pm 13.4 \mathrm{mmHg} \quad(P<0.01)$; diastolic BP from $68.9 \pm 7$ to $77 \pm 10 \mathrm{mmHg}$ $(P<0.01)$. In group B mean systolic BP varied from $112.1 \pm 16.3 \mathrm{mmHg}$ in basal conditions to $167.5 \pm 24.0$ during cystometry $(P<0.01)$ and diastolic BP from $72.9 \pm 10.5$ to $101.8 \pm 8.8(P<0.001)$. BP increase in group $\mathrm{B}$ was higher than $\mathrm{BP}$ variations in non $\mathrm{AD}$ patients (systolic BP $167.5 \pm 24.0 \mathrm{mmHg} \quad v s$ $121.6 \pm 13.4, \quad P<0.01$; diastolic $101.8 \pm 8.8 \mathrm{mmHg}$ vs $77.0 \pm 10.3, P<0.01)$.

$\mathrm{PR}$ was $76.3 \pm 16.3$ beat per minute in group $A$ at the beginning of the examination and $69.8 \pm 14.3$ at the end; in group B it was 74.4 \pm 17.5 and $64.9 \pm 19.7$. Seven patients in group A and five in group B showed an increase of PR during bladder voiding, meanwhile 13 patients in group A and 10 in group B showed a decreased; in the remaining patients in the two groups the PR was stable during the all examination. The differences between the two groups regarding $\mathrm{PR}$ were not statistically significant.

Thirteen patients in group B $(65 \%)$ showed both AD symptoms and BP increase, the other seven $(35 \%)$ only $\mathrm{BP}$ increase. In three patients $\mathrm{BP}$ increase began when uninhibited contraction started, in 11 it was coincident with uninhibited contraction peak and in the other six it appeared at maximum bladder capacity.

AD was more frequent in cervical patients $(P<0.05)$. There was no correlation of AD with other clinical features (sex ratio, age, disease duration, completeness of lesion), incidence of detrusor hyperreflexia/areflexia and detrusor-sphincter dyssy-

Table 1 Clinical features of the patients

\begin{tabular}{lccc}
\hline & Group A & Group B & $p$ \\
\hline Sex ratio (M/F) & $24 / 4$ & $18 / 2$ & n.s. \\
$\begin{array}{l}\text { Age (years) (mean + SD) } \\
\text { Disease duration }\end{array}$ & $30.9 \pm 12.4$ & $31.1 \pm 10.4$ & n.s. \\
$\quad$ (months) (mean + SD) & $41.9 \pm 66$ & $57.8 \pm 75.6$ & n.s. \\
$\begin{array}{c}\text { Lesional level (cervical/ } \\
\text { thoracic) }\end{array}$ & $11 / 17$ & $13 / 7$ & 0.034 \\
$\begin{array}{c}\text { Completeness of lesion } \\
\text { (complete/uncomplete) }\end{array}$ & $5 / 23$ & $4 / 16$ & n.s. \\
\hline
\end{tabular}

nergia (Tables 1 and 2). In fact in our series two patients showed AD although they had detrusor areflexia; in these case AD crisis began at maximum bladder distension.

With regard to voiding modalities the two groups did not show any significant difference although patients in group A voided more regularly by means of intermittent catheterization.

No statistically significant differences were found with regard to therapy with anticholinergic drugs: in fact 11 patients in group A were under therapy $v s$ seven in group B $(P=0.942)$ and mean duration of treatment was $8.4 \pm 7.6$ months in group $A$ and $10.8 \pm 7.5$ months in group B $(P=0.477)$.

Patients on anticholinergic treatment showed a better urodynamic picture than patients without therapy: uninhibited contractions threshold: $290.7 \mathrm{ml}$ vs $159.7 \mathrm{ml} \quad(P=0.195)$, uninhibited contractions amplitude: 53.3 vs $94.8 \mathrm{cmH}_{2} \mathrm{O} \quad(P<0.05)$, bladder capacity: 371.4 vs $309.8 \mathrm{ml}(P=0.384)$; nevertheless in these patients autonomic dysreflexia parameters are not significantly different.

\section{Discussion}

Autonomic dysreflexia following spinal cord lesion was first reported at the end of nineteenth century. In 1947 Guttman and Whitteridge ${ }^{12}$ reported the relationship between autonomic dysreflexia and bladder distension.

The pathophysiology of AD is the following: proprioceptive and nociceptive impulses from the bladder (detrusor contraction or bladder distension) ascend along the afferent pathways (pelvic and hypogastric nerves) in the spino-thalamic tracts and posterior columns; the efferent reflex outflow from the lateral horns of the spinal cord through the sympathetic pathways causes spasm of the pelvic viscera, arterial spasm with blood pressure increased, piloerection and sweating. ${ }^{13}$ In normal subjects the activation of baroreceptors, through the vagus nerve and descending pathways to the spinal cord, causes bradycardia and vasodilatation. In patients with a lesion above the level of the splanchnic outflow inhibitory impulses cannot reach the effector organs resulting in autonomic dysreflexia. ${ }^{1}$

Our results show the presence of autonomic dysreflexia during urodynamics in $41.6 \%$ of our patients; of these patients seven $(35 \%)$ do not show

Table 2 Urodynamic features

\begin{tabular}{|c|c|c|c|c|}
\hline Mean & Group $A+$ Group $B$ & Group $A$ & Group $B$ & $p$ \\
\hline DH (No.) & 3 & 2 & 1 & 0.762 \\
\hline DA (No.) & 6 & 4 & 2 & 0.574 \\
\hline $\mathrm{DH}+\mathrm{DSD}($ No.) & 39 & 22 & 17 & 0.573 \\
\hline Low compliance (No.) & 9 & 5 & 4 & 0.348 \\
\hline Uninhibited contractions threshold (ml) & $253(47-480)$ & 284 & 220 & 0.234 \\
\hline Uninhibited contractions amplitude (cmH2O) & $74(20-170)$ & 71 & 78 & 0.545 \\
\hline Capacity (ml) & $363(47-500)$ & 341 & 394 & 0.447 \\
\hline
\end{tabular}


symptoms or awareness of blood pressure increase. According to other studies this finding confirms the presence of silent autonomic dysreflexia and suggests that the absence of symptoms cannot be considered an exclusion criteria of the disease. ${ }^{4}$ The finding of silent dysreflexia could be explained by the fact that symptoms and signs in these patients are too weak to be recognised by the patients themselves or by a mechanism of adjustment.

In our series also the patients without dysreflexia showed a statistically significant increase of both diastolic and systolic blood pressure. Four of these patients $(10.2 \%)$, all with a complete clinical lesion, had clinical signs of autonomic dysreflexia too. According to these data autonomic dysreflexia could be considered a gradual reaction ranging from non dangerous sympathetic stimulation to full AD crisis. These findings may be explained by the variable extension and completeness of the anatomical lesion, with variable responses to bladder filling and voiding.

In our patients $\mathrm{AD}$ presence is significantly more frequent in patients with lesions at the cervical lesion, probably because the splanchnic outflow is completely free from inhibitory regulation. On the contrary it is not related to other clinical parameters (age, sex, completeness of lesion, distance from injury and bladder management).

With regard to urodynamic data, in our series only three patients showed autonomic dysreflexia at the beginning of uninhibited detrusor contractions, while 11 patients had dysreflexia during uninhibited detrusor contractions; in the remaining six patients AD appeared at maximum bladder capacity, without any temporal relationship with uninhibited detrusor contractions. The relationship between autonomic dysreflexia and bladder distension or uninhibited detrusor contractions is not completely clear. Per$\mathrm{kash}^{14}$ found a relationship between autonomic dysreflexia and detrusor-sphincter dyssynergia in tetraplegic patients. In these patients bladder neck and posterior urethra compliance could be reduced, causing an increased stretching of these structures and an increased receptor and afferent pathways activation resulting in autonomic dysreflexia. Furthermore the frequency and severity of autonomic dysreflexia responses were significantly reduced by external sphincterotomy ${ }^{5,15-17}$ or by the administration of small doses of an alpha-adrenergic-blocker. ${ }^{17}$

Linsemeyer ${ }^{4}$ emphasises that blood pressure increase in patients with uninhibited detrusor contractions is not caused by bladder distension itself, but by bladder distension triggering uninhibited bladder contraction. Finally Thyberg ${ }^{6}$ found a relationship between autonomic dysreflexia and detrusor uninhibited contractions duration.

Our results show that autonomic dysreflexia could be due to both the presence of uninhibited contractions and bladder distension. Thus, bladder distension remains an important factor determining, at adequate volumes, a stimulation of detrusor tension receptors; this could also explain the finding of symptoms and signs of autonomic dysreflexia in patients with detrusor areflexia due to spinal shock. In these patients autonomic dysreflexia could be the first sign of spinal shock recovery.

It is worth noting that patients with autonomic dysreflexia showed lower threshold and higher amplitude of uninhibited detrusor contractions when compared to patients with non-dangerous blood pressure increase, but these differences were not statistically significant. Furthermore, in our series seven patients in group B were on anticholinergic treatment and showed a better urodynamic picture with particular regard to amplitude of uninhibited detrusor contractions; nevertheless autonomic dysreflexia parameters (diastolic and systolic pressure and heart rate) were comparable with those of non-treated patients. These data could confirm that uninhibited detrusor contractions are not the only trigger factor of autonomic dysreflexia.

Treatment with anticholinergic drugs is not sufficient to prevent autonomic dysreflexia starting from the bladder, unless it induces detrusor areflexia. Also in this case patients are at risk of developing autonomic dysreflexia following bladder distension.

\section{Conclusions}

During urodynamic evaluation all the patients with a lesion level above T6 showed significant blood pressure increase if compared to basal condition, although only $20(41.6 \%)$ showed dangerous values.

The relationship between urodynamic data and dysreflexia crisis shows that both the presence of detrusor uninhibited contractions and bladder distension are able to stimulate the crisis. Autonomic dysreflexia may be present also in patients with detrusor areflexia in the spinal shock phase; in this case it may represent one of the first signs of recovery from the shock itself.

Treatment with anticholinergic drugs is not sufficient to prevent autonomic dysreflexia starting from the bladder, unless it induces detrusor areflexia. Also in this case patients are at risk of developing autonomic dysreflexia following bladder distension.

Patients and physicians should thus be aware of the possibility of an autonomic dysreflexia crisis; patients should be submitted to regular blood pressure and urodynamic controls to establish adequate pharmacological treatment and bladder emptying regimen.

\section{References}

1 Kurnick NB. Autonomic hyperreflexia and its control in patients with spinal cord lesions. Ann Int Med 1956; 44: 678-683.

2 Erickson RP. Autonomic hyperreflexia: pathophysiology and medical management. Arch Phys Med Rehab 1980; 61: 431 - 434

3 Lindan R, Joiner E, Freehafer AA, Hazel C. Incidence and clinical features of autonomic dysreflexia in patients with spinal cord injury. Paraplegia 1980; 18: $285-289$. 
4 Linsenmeyer TA, Campagnolo DI, Chou I. Silent autonomic dysreflexia during voiding in men with spinal cord injuries. J Urol 1996; 155: $519-522$.

5 Ricottone AR, Pranikoff K, Steinmetz JR, Costantino G. Longterm follow-up of sphincterotomy in the treatment of automonic dysreflexia. Neurourology and Urodynamics 1995; 14: 43-46.

6 Thyberg M, Ertzgaard P, Gylling RN, Granerus G. Effect of nifedipine on cystometry-induced elevation of blood pressure in patients with a reflex urinary bladder after a high level spinal cord injury. Paraplegia 1994; 32: $308-313$.

7 Di Tunno JF, Young W, Donovan WH, Creasey G. The international standards booklet for neurological and functional classification of spinal cord injury. Paraplegia 1994; 32: 70-80.

8 Abrams P, Blaivas JG, Stanton SL, Anderson JT. The standardization of terminology of lower urinary tract function. The international Continence Society Committee on standardization of terminology. Scand J Urol Nephrol 1988; 114: 5-19.

9 Blaivas JG and Barbalias GA. Detrusor-external sphincter dyssynergia in men with multiple sclerosis: an ominous urological condition. J Urol 1984; 131: 91-94.

10 Guttman L, Frankel HL, Paeslack V. Cardiac irregularities during labor in paraplegic women. Paraplegia 1965; 3: $144-151$.
11 Acute management of Autonomic dysreflexia: Adults with Spinal Cord Injury Presenting to Health Care Facilities. Published by Paralyzed Veterans of America Consortium for Spinal Cord Medicine, Washington, 1997.

12 Guttmann L, Whitteridge D. Effects of bladder distension on autonomic mechanism after spinal cord injuries. Brain 1947; 70: $361-404$.

13 Erickson RP. Autonomic hyperreflexia: pathophysiology and medical management. Arch

14 Perkash I. Pressor response during cystomanometry in spinal cord injured patients complicated with detrusor-sphincter dyssynergia. J Urol 1997; 121: 778 - 784.

15 Perkash I. Detrusor-sphincter dyssynergia an dyssynergic responses: recognition and rational for early modified transurethral sphincterotomy in complete spinal cord lesions. J Urol 1978; 120: $469-474$.

16 Barton $\mathrm{CH}$ et al. The effects of modified transurethral sphincterotomy on autonomic dysreflexia. J Urol 1986 135: $83-85$.

17 McGuire EJ, Rossier AB. Treatment of acute autonomic dysreflexia. J Urol 1983 129: 1185-1189. 